\title{
Proceedings of the 24th International Stroke Genetics Consortium Workshop: Washington, DC, USA
}

Neurol Genet 2019;5:e325. doi:10.1212/NXG.0000000000000325

\section{24th Workshop of the International Stroke Genetics Consortium, November 8-9, 2018, Washington, DC, USA}

Paul A. Nyquist, MD, $M P H^{1}$ and John W. Cole, $M D, M S^{2}$

${ }^{1}$ Department of Anesthesiology and Critical Care Medicine, The Johns Hopkins Hospital, Baltimore, $\mathrm{MD} ;{ }^{2}$ Department of Neurology, Baltimore VA Medical Center and University of Maryland School of Medicine, Baltimore.

The 24th workshop of the International Stroke Genetics Consortium (ISGC) was held at the beautiful National Museum of the American Indian on November 8-9, 2018 in Washington, DC, and was hosted by Paul Nyquist, MD, MPH and John Cole, MD, MS, and co-hosted by Jemma Hopewell, $\mathrm{PhD}$ and Matthew Traylor, $\mathrm{PhD}$. The ISGC is an international collaboration of physicians and scientists who have agreed to pool resources and expertise in an effort to unravel the genetic basis of stroke and its comorbidities. It was formed in 2007 by a small group of scientists and has since grown to over 200 members representing over 50 countries in North and South America, Europe, Australia, Africa, and Asia. ISGC workshops are held semi-annually and provide a forum for ISGC scientists and researchers to report on progress of ongoing scientific projects and discuss new ideas that help advance the ISGC's research mission. The ISGC nurtures its junior members by offering several travel awards to young investigators. Many abstracts in these Proceedings reflect the contributions of active junior investigators. Herein, we present the Proceedings and official published abstracts of the 24th ISGC workshop.

Presentations at the workshop included invited lectures, ISGC working group reports, and short presentations about ongoing research projects and new collaborative proposals. This particular workshop benefited from its close proximity to the National Institutes of Health (NIH), with several presentations by senior NIH officials including the keynote address delivered by Walter Koroshetz, MD, the Director of the National Institute of Neurological Disorders and Stroke (NINDS). Major themes of the 24th ISGC workshop revolved around the analysis of large, multi-ethnic samples to identify novel stroke genes; the genetics of stroke outcome; patient advocacy; and the integration of functional genomic approaches to human genetic studies to define the biological mechanisms underlying stroke risk. Notably, several premeetings occurred focusing on the genetics of young-onset stroke; the genetic basis of whitematter hyperintensities; and future ISGC plans for a large collaborative study evaluating the genetics of stroke outcome. The ISGC actively invites other like-minded collaborative scientists to join, with our next Workshops occurring in Cambridge, UK in April 2019 and Saint Louis, Missouri, USA in November 2019. We encourage you to visit our website (strokegenetics.org) for further details.

On behalf of the ISGC Steering Committee and its many members, we hope that you enjoy reviewing these Proceedings.

Acknowledgment: Steering Committee-Stephanie Debette, Chair and Jin-Moo Lee, Vice Chair; Data Access Committee-Jemma Hopewell; Analysis Committee-Rainer Malik; Outreach Committee-Jenny Majersik; Acute Endophenotypes Working Group-Jin-Moo Lee and Israel Fernandez; Imaging Working Group-Natalia Rost; Outcomes Working 
Group-Arne Lindgren; Intracerebral Hemorrhage Working Group-Jonathan Rosand and Daniel Woo; Intracranial Aneurysm Working Group-Ynte Ruigrok; Translational Science Working Group-Chris Anderson; Biobank Access Working Group-Jemma Hopewell.

Correspondence Dr. Cole jcole@som.umaryland.edu

\section{The role of mitochondrial DNA} variations on intracranial atherosclerosis in Han Chinese patients

Aaron M. Gusdon, $M D,{ }^{1}$ Ruinan Shen, $M D,{ }^{2}$ Ying Tan, $M D,{ }^{3}$ Jianqing Ding, $M D,{ }^{2}$ Bruce Wasserman, $M D,{ }^{4}$ Paul Nyquist, $M D, M P H,{ }^{1}$ and Sheng-di Chen, $M D^{2}$

${ }^{1}$ Division of Neurocritical Care, Department of Neurology, Johns Hopkins University, Baltimore, MD; ${ }^{2}$ Department of Neurology, Shanghai Jiaotong University, Shanghai, China; ${ }^{3}$ Department of Neurology, Xi'an Jiaotong University, Xi'an, China; ${ }^{4}$ Department of Radiology, Johns Hopkins University, Baltimore, MD.

Objective To determine whether mitochondrial DNA (mtDNA) variants contribute to the development of intracranial atherosclerosis. Background Intracranial atherosclerosis contributes to stroke by causing occlusions of large intracranial arteries often by plaque rupture. Intracranial atherosclerosis accounts for a higher proportion of ischemic strokes in Asian populations. While several nuclear genes have been implicated in large vessel disease, with varying results in Eastern and Western populations. Importantly, East Asian populations harbor distinct mtDNA variations. The C5178A polymorphism, defining mitochondrial haplogroup $\mathrm{D}$, has been shown to decrease the risk of several age-related conditions and reduces reactive oxygen species (ROS) production. We hypothesized that distinct mtDNA variations would contribute to intracranial atherosclerosis in East Asian patients and these variants would modify the pathogenicity of nuclear loci. Design/Methods We prospectively enrolled 437 Han Chinese patients admitted with stroke in 2 Chinese cities, Shanghai and Xi'an. MRI and MRA were performed as part of the stroke workup. mtDNA was amplified in 4 segments and sequenced. Mitochondrial SNPs were determined using MitoMaster. Large vessel disease was assessed qualitatively using WASID criteria and quantitatively using a semi-automated algorithm. Small vessel disease (SVD) was quantified using a scoring system accounting for white matter hyperintensities (Fazekas), microbleeds, and lacunes. Results Among 437 patients, 132 encoded C5178A (30.2\%). There was an inverse correlation between degree of intracranial atherosclerosis and C5178A (beta $-12.2, p<0.001$ ). A similar correlation was noted between D sub-haplogroups. No association was found between any mitochondrial haplogroup and the degree of SVD. Work is currently underway genotyping nuclear loci known to contribute to intracranial atherosclerosis in order to determine if they are modulated by mtDNA variants. Conclusions The mtDNA variant C5178A was associated with less large vessel intracranial atherosclerosis without affecting the degree of SVD. This may be due to decreased ROS production. Future work will focus on elucidating the interactions between nuclear and mtDNA loci.

Contact Email: agusdon1@jhmi.edu

Disclosure and Study Support: This study was supported by a grant from the National Natural Science Foundation of China.

\section{Improved genomic coverage of the SiGN dataset: Imputation using TOPMED vs HRC reference panels}

T.J. Jaworek, BS, ${ }^{1}$ B.J. Gaynor, BS, ${ }^{1}$ K.A. Ryan, MS, ${ }^{1}$ B.D. Mitchell, $P h D,{ }^{1,2}$ and H. Xu, MD, $P h D^{1}$

${ }^{1}$ University of Maryland, Department of Medicine, Baltimore, $\mathrm{MD} ;{ }^{2}$ Geriatric Research, Education and Clinical Center, Veterans Affairs Medical Center, Baltimore, MD.

Objective We aim to evaluate the effects of using the newest TOPMED imputation reference panels on imputation quality for studies of stroke genetics. Background Modern genomewide association studies (GWAS) rely on high density SNP arrays and imputation to increase coverage of the genome. Recently, the Haplotype Reference Consortium (HRC) genomes have replaced 1000 Genomes (1000G) as the standard imputation reference panel for European Caucasian (EUR) samples due to denser variant coverage and more haplotypes included (39.2M variants, 64,976 haplotypes). The TOPMED WGS data Freeze 5 includes $463 \mathrm{M}$ variants that define 125,568 haplotypes. We hypothesize that re-imputing array data with the TOPMED reference panel will increase the imputation accuracy and genomic coverage for European Caucasian samples compared to using HRC. Design/Methods EUR samples in the Stroke Genetics Network (SiGN) genotyped on the Illumina $5 \mathrm{M}$ array $(\mathrm{n}=38,220)$ were uploaded to the Michigan Imputation Server (imputationserver.sph.umich. edu/) following QC for imputation against both the HRC (version r1.1) and TOPMED (freeze 5) panels. Eagle v2.4 and Minimac 3 were used for Phasing and imputation, respectively. We assessed imputation quality by analyzing quality metrics (R2) and genotypic concordance with directly genotyped markers. Results Samples of European ancestry genotyped on the Illumina $5 \mathrm{M}$ array demonstrated overall high-quality imputations with both panels. Compared to HRC based imputation, the TOPMED panel resulted in a $45.4 \%$ increase in the number of high-quality imputed variants $(19.4 \mathrm{M}$ vs $13.3 \mathrm{M}$ ), with most of the power gain occurring for imputed rare variants with $\mathrm{MAF}<1 \%$ ( $12.9 \mathrm{M}$ vs $7.6 \mathrm{M}, 68.4 \%$ increase). 
At the individual variant level, there were $12,061,322$ overlapping variants between these 2 imputation outputs. However, 7,246,653 variants were present only in TOPMED based imputation vs $1,236,244$ variants uniquely imputed with HRC panel. Conclusions The use of TOPMED reference panel for GWAS imputation substantially increases genomic coverage for rare variants compared to HRC reference-based imputation. TOPMED could potentially become a new standard resource to meet the needs of GWAS imputation.

Contact Email: tjaworek@som.umaryland.edu

Disclosure and Study Support: Supported by NIH grants (R01NS100178 and T32AG000262).

\section{The promise of standardized biobanking in regenerative medicine}

D.A. Taylor, PhD, Hon DSc, ${ }^{1}$ K. Kirkwood, $M S,{ }^{2}$ L.C. Sampaio, $M D, M S^{1}$; on Behalf of the CTSN Biorepository Committee

${ }^{1}$ Regenerative Medicine Research, Texas Heart Institute, Houston; ${ }^{2}$ Icahn School of Medicine at Mount Sinai, New York.

Objective To describe how biobanking and profiling of biospecimens linked to clinical data presents a unique opportunity to study the safety, efficacy, and impact of regenerative medicine products after ischemic injury. Background Regenerative medicine seeks to treat the underlying injury rather than the symptoms associated with an injury. Most regenerative medicine products are complex and heterogeneous biologics (e.g., cells and cell secretomes) rather than being a single homogeneous entity like a drug. Moreover, the product may be present and active long term and may cause a systemic response in addition to affecting the target. By linking stored biospecimens to patient-level clinical and demographic information, it is possible to explore common pathways of disease and response to treatment, providing crucial mechanistic insights into these novel therapies, as well as developing a more accurate safety profile of the products. We have recently shown that after acute myocardial infarction, the presence of specific cells in bone marrow or blood associate with improvement irrespective of treatment. Ischemic stroke shares many commonalities with myocardial infarction. Design/Methods Profiling extremes is one method to define targets applicable to a wide spectrum of studies in regenerative medicine. Paired samples of biospecimens (e.g., tissue, blood) are collected from each patient before and after treatment. After treatment, patients are ranked in terms of outcome improvement, defined by a given trial's primary outcome measure, irrespective of treatment group (improvers/non-improvers) and in response to treatment (responders/non-responders). The top 15\% and bottom 15\% of patients ("responders" and "non-responders") are selected and analyzed for differences in biospecimens (e.g., growth factors, chemo/cytokines, microRNAs, proteins, etc.). Results
We have previously shown this method can be applied in cardiovascular cell therapy studies (Taylor et al., PMID 26590374) to define cell profiles associated with both improvers. We showed that the frequency of hematopoietic $\left(\mathrm{CD} 19^{+}, \mathrm{CD}_{11 \mathrm{~b}^{+}}\right)$, immune $\left(\mathrm{CD} 19^{+} \mathrm{CXCR} 4^{+}\right)$, and migratory (CD11b and CXCR4) bone marrow cells associated with positive outcome in cell therapy patients with chronic ischemic LV dysfunction and have expanded these results to acute MI. Identifying similar associations in patients with thromboembolic events could provide novel targets for therapy. CTSN has access to numerous patients at risk for or having had thrombo-embolic injury secondary to cardiac surgery and/or atrial fibrillation with linked clinical, demographic, and biospecimen data, providing a unique opportunity for these novel analyses. Conclusions In conclusion, the combination of biospecimen data with clinical and demographic information can lead to important insights about disease progression and treatment response, leading to the development of novel treatment strategies and the ability to determine which patients are most likely to benefit from existing treatment strategies. Standardized nomenclature, definitions, and assays are vital to linking data across studies.

Contact Email: dtaylor@texasheart.org

Disclosure and Study Support: None.

\section{The second phase GISCOME initiative: A genome-wide association meta-analysis of functional outcome after ischemic stroke}

C. Lagging $M D,,^{1^{*}}$ M. Söderholm, MD, PhD,,$^{2^{*}}$ A. Pedersen, $M D,{ }^{1}$ E. Lorentzen, $M S c^{3}{ }^{3}$ T.M. Stanne, PhD, ${ }^{1}$ J.M. Maguire, RN, $\mathrm{PhD},{ }^{4+}$ A. Lindgren, $\mathrm{MD}, \mathrm{PhD},{ }^{2+}$ and C. Jern, $\mathrm{MD}, \mathrm{PhD}^{1+}$; on behalf of the International Stroke Genetics Consortium and GISCOME network

${ }^{1}$ Inst of Biomedicine, University of Gothenburg, Gothenburg, Sweden; ${ }^{2}$ Dept of Clinical Sciences Lund, Lund University, Lund, Sweden; ${ }^{3}$ Bioinformatics Core Facility, University of Gothenburg, Gothenburg, Sweden; ${ }^{4}$ University of Technology Sydney, Faculty of Health, Sydney, Australia.

${ }^{*}$ These authors contributed equally to this work.

${ }^{+}$These authors jointly supervised this work.

Objective To discover common genetic variants associated with post-stroke functional outcomes. Background It is likely that genetic factors account for a part of the large interindividual variability in recovery after ischemic stroke. The first genome-wide association (GWA) study on functional outcome performed within the Genetics of Ischaemic Stroke Functional Outcome (GISCOME) network has been completed. It 
comprised approximately 6,000 participants with ischemic stroke of predominantly European ancestry. One variant with genome-wide significance and several variants with suggestive association to functional outcome were identified. These results need replication, and a larger sample is needed in order to identify novel variants. We therefore propose a second phase of the GISCOME initiative. Design/Methods This proposed meta-analysis will comprise data from all cohorts in the existing GISCOME GWA study and from additional cohorts of ischemic stroke participants with data on modified Rankin Scale (mRS) score 60-190 days after stroke and covariates including age, sex, and stroke severity (baseline NIH Stroke Scale score). Ideally, genotyping is completed or alternatively, cohorts will have DNA available for genotyping. Data on stroke subtype, infarct location, reported ancestry, acute treatments and comorbidities will be collected when available. The same analysis plan as in GISCOME phase 1 will be applied, i.e., mRS will be analyzed both as a dichotomous and ordinal variable. We aim to perform subgroup analyses according to infarct location and selected comorbidities such as diabetes. Further, we invite participants from all continents and aim to study associations in different ancestry groups. Results Not applicable. Conclusions We propose an extended GWA study of functional outcome after ischemic stroke, and we invite new centers to join the GISCOME-collaboration in the search for genetic factors influencing post-stroke outcomes.

Contact Email: cecilia.lagging@gu.se

Disclosure and Study Support: Swedish Research Council, Swedish Heart and Lung Foundation, Swedish State; ALF agreement, Swedish Stroke Association, Australian National Health and Medical Research Council. The authors report no disclosures.

\section{Seven loci identified in epigenome-} wide survival analysis of time to recurrent stroke, composite vascular endpoint, and disabling/fatal stroke, myocardial infarction or death in participants from the VISP clinical trial

Nicole M. Davis Armstrong, PhD, ${ }^{1}$ Wei-Min Chen, PhD, ${ }^{2}$ FangChi Hsu, PhD, ${ }^{3}$ Michael S. Brewer, PhD, ${ }^{1}$ Stephen R. Williams, $\mathrm{PhD},{ }^{2}$ Michele M. Sale, PhD, ${ }^{2}$ Bradford B. Worrall, $M D,{ }^{2}$ and Keith L. Keene, $P h D^{3}$

${ }^{1}$ East Carolina University, Greenville, NC; ${ }^{2}$ University of Virginia, Charlottesville; ${ }^{3}$ Wake Forest School of Medicine, Winston-Salem, NC.

Objective We performed survival analyses using Cox proportional hazards models to test for associations between the degree of methylation and time to recurrent/coronary endpoints. Background Recurrent strokes account for approximately $25 \%$ of all strokes annually in the US and are more-deadly and -disabling than initial stroke. Unfortunately, recurrent stroke is a vastly understudied phenotype with limited genetic, epigenetic, and epidemiologic data. Design/Methods Using Illumina HumanMethylation450K arrays, $\beta$-values were generated using DNA extracted from whole blood samples of 180 participants from the Vitamin Intervention for Stroke Prevention (VISP) clinical trial. The $\beta$-values for 473,864 autosomal $\mathrm{CpG}$ loci were analyzed using models adjusted for covariates and stratified by ethnicity: 76 of African descent (AFR) and 104 of European descent (EUR). The endpoints included VISP recurrence (stroke that occurred during VISP), composite vascular (bypass graft, MI, angioplasty, cardiac resuscitation, fatal coronary endpoint, or recurrent stroke during VISP), and severe stroke/MI ("FDMD", disabling/ fatal stroke or MI, or death during VISP). Results In total, 7 loci reached or exceeded genome-wide significance ( $p$ less than or equal to $1.05 \mathrm{e}-07)$ and 353 loci reached our suggestive threshold ( $p$ less than or equal to $5 \mathrm{e}-06$ ). The most significant association was observed for $\mathrm{cg} 04059318$ (AFR VISP Recurrent; $p=4.52 \mathrm{e}-08$ ), which is located in a binding site for PTEN. Neuronal PTEN loss results in neuroprotection against ischemia acutely but contributes to vascular cognitive impairment long term. cg00076998 (AFR composite; $p=7.87 \mathrm{e}-08$ ) is located within a binding site for PIK3CB. PIK3CB has been described to mediate pulmonary vascular smooth muscle proliferation. cg02365967 (EUR composite; $p=8.08 \mathrm{e}-08$ ), is located in exon 35 of HERC2, which has been implicated in the regulation and prevention of adverse arterial remodeling. Conclusions In conclusion, our survival analyses identified 7 statistically significant and 353 suggestively significant associations between the degree of methylation and time to VISP recurrent stroke, composite vascular endpoint, or severe stroke/MI endpoint, with several loci located within gene regions having plausible stroke and cardiovascular implications.

Contact Email: davisnic12@students.ecu.edu

Disclosure and Study Support: AHA Grant (12SDG9180012) and Walking for My Life 5K Stroke Walk sponsored by Faith Christian Center International.

\section{Beyond the brain: A systematic} review of phenotypes associated with mutations in mendelian cerebral small vessel disease genes

David Henshall, PhD, ${ }^{*}$ Isaac Kuan, PhD, ${ }^{*}$ Blair Wilson, $M D$, Cathie Sudlow, $M D{ }^{+}$and Kristiina Rannikm, $M D^{+}$

University of Edinburgh, Edinburgh, UK.

*/ + : these authors contributed equally. 


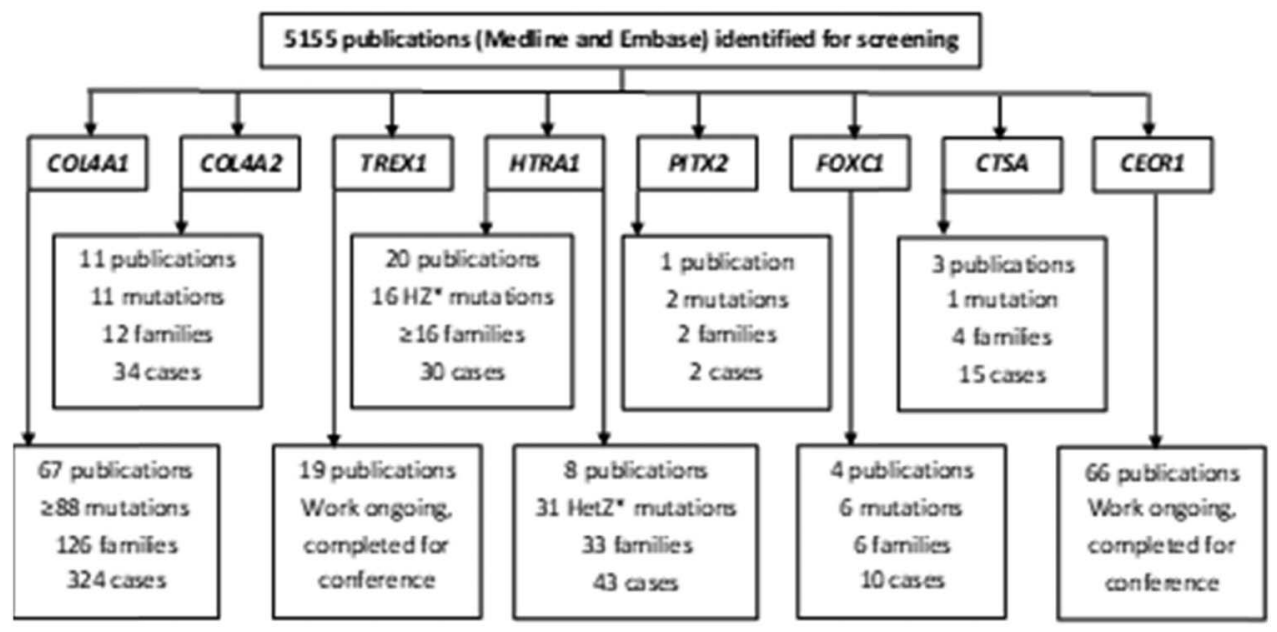

"HZ: homazygous or campound heterozyous; HetZ: heterozygous

Objective To identify and quantify systemic phenotypes associated with Mendelian cerebral small vessel diseases (CSVD) to improve our understanding of underlying CSVD mechanisms and improve diagnosis of Mendelian forms. Background CSVD accounts for $20 \%$ of strokes and causes disability and cognitive decline. The majority of CSVD is sporadic, but rarely it can occur due to single gene mutations. It is increasingly recognized that these Mendelian diseases have clinical manifestations beyond CSVD. Design/Methods We: (1) systematically sought publications of cases with mutations in known Mendelian CSVD genes: COL4A1/2, TREX1, HTRA1, PITX2, FOXC1, CTSA, CECR1; (2) extracted the number of reported unique mutations, families and cases, and their age and sex; (3) extracted and quantified reported systemic phenotypes for each gene. Results We included 198 of 5155 publications screened (figure 1). Where reported, median age of cases ranged from 17 years (COL4A2) to 61 years (HTRA1 heterozygotes), and 30\% (HTRA1 heterozygotes) to 87\% (CTSA) were female. In addition to CSVD, the most commonly involved organ-systems and phenotypes were: eye (40\% COL4A1, 3\% COL4A2, 100\% PITX2, 90\% FOXC1), kidneys (16\% COL4A1, 3\% COL4A2), muscle (18\% COL4A1, 6\% COL4A2), premature hair loss (83\% HTRA1 homozygotes, $16 \%$ heterozygotes), spondylosis (90\% HTRA1 homozygotes, $42 \%$ heterozygotes), depression (0.3\% COL4A1, 3\% HTRA1 homozygotes, 9\% heterozygotes, 46\% CTSA), migraine (5\% COL4A1, 3\% COL4A2, 40\% CTSA, 5\% HTRA1 heterozygotes) and sensorineural hearing loss $(0.6 \%$ COL4A1, 7\% CTSA, 10\% FOXC1, 3\% HTRA1 homozygotes and $2 \%$ heterozygotes). Work is ongoing for TREX1 and CECR1 genes. Conclusions In conclusion, manifestations beyond CSVD are very common and demonstrate the systemic nature of these Mendelian diseases.

Contact Email: kristiina.rannikmae@ed.ac.uk

Disclosure and Study Support: No disclosures.

\section{Japan collaborative project on intracranial aneurysm}

\author{
Hirofumi Nakatomi, MD, PhD, ${ }^{1}$ UCAS Study Group University \\ of Tokyo Study Group \\ ${ }^{1}$ University of Tokyo, Department of Neurosurgery.
}

Objective The goal of this study is to summarize the current status of collaborative national, intra-college and inter-college clinical cohort studies on intracranial aneurysms in Japan. Background The natural history (genesis, progression, rupture and healing) of intracranial aneurysms including saccular aneurysms, acute intracranial dissection and chronic fusiform \& dolichoectatic aneurysms are still unknown. What phenotypic and genotypic factors determine the diversity and aggressiveness of these intracranial aneurysms are also still uninvestigated. Design/Methods We have performed multicenter prospective cohort studies for unruptured cerebral aneurysms as UCAS, for intracranial arterial dissections as University of Tokyo Study (IAD-UTS) and for giant/large intracranial fusiform and dolichoectatic aneurysms of vertebrobasilar junction and basilar trunk as Inter-college megadolicho basilar study (ICMBS). Results UCAS demonstrated that during a follow-up period that included 11,660 aneurysmyears, ruptures were documented in 111 patients, with an annual rate of rupture of $0.95 \%$. The risk of rupture varies according to size, specific site, and shape. IAD-UTS of 143 patients with a mean follow-up of 8.2 years revealed that IAD is a disease carrying a relatively high risk of symptomatic recurrence, apparently occurring in 3 phases and patterns: early hemorrhagic recurrence, late non-hemorrhagic recurrence, and chronic fusiformaneurysm transformation. ICMBS showed of untreated 11 patients, 10 died around 5 years, and one remained severely disabled. Twenty-one surgically treated patients had significantly longer overall survival 
compared with those who received conservative therapy. Conclusions Natural history of unruptured cerebral aneurysms, intracranial arterial dissections and intracranial dolichoectasia varies profoundly. Future genotype-phenotype international collaborative studies are needed to improve the treatment and their outcome.

Contact Email: hirofuminakatomi@gmail.com

Disclosure and Study Support: No disclosure. Study supported by Japanese Ministry of Health, Labor and Welfare.

\section{Opportunities for mechanistic studies of stroke in collaboration with the Cardiothoracic Surgical Trials Network}

Katherine A. Kirkwood, MS, ${ }^{1}$ Steven R. Messe, $M D,{ }^{2}$ Doris A. Taylor, $\mathrm{PhD}^{3}$; on behalf of the Cardiothoracic Surgical Trials Network

${ }^{1}$ Icahn School of Medicine at Mount Sinai, New York; ${ }^{2}$ University of Pennsylvania School of Medicine, Philadelphia;

${ }^{3}$ Texas Heart Institute, Houston.

Objective To describe the Cardiothoracic Surgical Trials Network (CTSN), its research, and opportunities for collaboration with the International Stroke Genetics Consortium (ISGC). Background Cardiovascular disease (CVD) is a leading cause of morbidity and mortality in the United States and worldwide. In response to the growing burden of CVD and the need for rigorous clinical evaluation of new treatments, the National Heart Lung and Blood Institute, along with the Canadian Institutes for Health Research and the National Institute of Neurological Disorders and Stroke, created the CTSN in 2007 to design, conduct, and analyze collaborative clinical trials that evaluate surgical interventions and management approaches for the treatment of CVD in adult patients. CTSN includes clinical sites enrolling patients throughout the US, Canada, and Europe, and is supported by a data and clinical coordinating center; a biorepository facility; and neurocognitive, imaging, and biospecimen core laboratories. Importantly, stroke is a known and feared complication of cardiac surgery, and CTSN has focused on neurologic outcomes of surgery as a core mission, in order to improve outcomes for patients. Design/Methods CTSN has completed 7 randomized controlled trials (RCTs) with nearly 3,500 patients focusing on ischemic mitral regurgitation, atrial fibrillation, advanced heart failure, and intra-operative embolic protection; biospecimens were collected and banked in 5 of these trials and are linked to clinical data. The results of these trials will be used to inform the design of up to 5 future clinical trials focused on management of the risk of perioperative and long-term neurologic events and outcomes. Results CTSN is currently planning trials of anticoagulation for post-operative atrial fibrillation and bioprosthetic valve surgery, neuroprotection in valve surgery, and novel left ventricular assist devices. The Network hopes to collaborate with the ISGC on design and analysis of genetic analyses related to stroke events in these populations. Conclusions In conclusion, CTSN has successfully completed multiple CVD RCTs including studies focused on stroke and neurologic outcomes of surgery. New trials in populations at high risk of stroke provide an ideal opportunity for collaboration with the ISGC.

Contact Email: katherine.kirkwood@mountsinai.org

Disclosure and Study Support: None.

\section{Power to detect associations with polygenic risk scores}

Brady J. Gaynor, BS, ${ }^{1}$ Sylvia Wassertheil-Smoller, $\mathrm{PhD},{ }^{2}$ Qibin $\mathrm{Qi}, \mathrm{PhD},{ }^{2}$ Rebecca D. Jackson, $\mathrm{MD},{ }^{3}$ Kathleen Ryan, $\mathrm{MPH},{ }^{1}$ Patrick F. McArdle, PhD,${ }^{1}$ John W. Cole, MD, ${ }^{1}$ Steven J. Kittner, $M D,{ }^{1}$ Jordan W. Smoller, MD, ScD,${ }^{4}$ Huichun Xu, MD, PhD, ${ }^{1}$ and Braxton D. Mitchell, $P h D^{1}$

${ }^{1}$ University of Maryland School of Medicine, Baltimore; ${ }^{2}$ Albert Einstein College of Medicine, Bronx, NY; ${ }^{3}$ Ohio State University Wexner Medical Center, Columbus, OH; ${ }^{4}$ Massachusetts General Hospital, Harvard Medical School, Boston.

Objective To determine the association of a polygenic risk score (PRS) for major depression disorder (MDD) with ischemic stroke using 2 different versions of a PRS, one based on the 2013 Psychiatric Genetics Consortium MDD GWAS (PGC) and the second based on the larger 2018 PGC MDD GWAS. Background PRS are often used to determine if the genetic risk of one trait is associated with a second trait. Many PRS are constructed to include SNPs identified from a GWAS. Power to detect associations of the PRS with a trait is a function of the robustness of the PRS, which is related to the sample size of the GWAS used to construct the PRS and the proportion of trait variation explained by the PRS. Design/Methods We calculated 2 MDD PRS based on summary GWAS results generated from the Psychiatrics Genetics Consortium (PGC). The first MDD PRS was calculated from the 2013 PGC GWAS, which included 9,240 MDD cases and 9,519 controls. The second MDD PRS was calculated from the 2018 PGC GWAS, which included 59,851 MDD cases and 113,154 controls. We then determined if these scores were associated with ischemic stroke in European Caucasian subjects from the SiGN study $(n=12,577$ stroke cases and 25,643 controls). The association between the MDD PRS and stroke was estimated by logistic regression. Results The 2013 depression PRS accounted for $0.6 \%$ of the variation in MDD and was very weakly associated with ischemic stroke. In contrast, the 2018 depression PRS 
accounted for $2.3 \%$ of the variation in MDD and was strongly associated with a higher risk of ischemic stroke overall ( $p=$ $4.4546 \mathrm{E}-06$; $\mathrm{OR}=1.05 ; 95 \% \mathrm{CI}=1.03-1.08)$, as well as with stroke due to small artery occlusion $(p=0.00024$; OR $=1.09$; $95 \% \mathrm{CI}=1.04-1.14)$ and large artery atherosclerosis $(p=$ 0.00154 ; OR $=1.07$; $95 \% \mathrm{CI}=1.03-1.12)$. Conclusions We have shown that ischemic stroke is strongly associated with an MDD PRS generated from the 2018 PGC GWAS, but not from the 2013 PGC GWAS. These results highlight the greater robustness of larger sized GWAS that are better able to discriminate between true positive and false positive associations. Failure to detect associations with PRS should not necessarily be interpreted as a lack of pleiotropy but could reflect low power.

Contact Email: bgaynor@som.umaryland.edu

Disclosure and Study Support: No disclosures. Supported by R01 NS100178.

\section{Mendelian randomization of thousands of blood biomarkers identifies potential causal mediators for ischemic stroke subtypes}

M. Chong, PhD, J. Sjaarda, PhD, M. Pigeyre, MD, PhD, P. Mohammadi-Shemirani, PhD, H. Gerstein, $M D, M S c$, and G. Pare, $M D, M S c$

Population Health Research Institute, Hamilton, ON, Canada.

Objective With the purpose of identifying novel targets for pharmacologic intervention, we conducted Mendelian Randomization (MR) analysis to discover causal blood biomarkers for ischemic stroke subtypes. Background Stroke is a leading cause of death and disability worldwide, and approximately 1 in every 4 strokes occur in patients who have already had a stroke event. Clearly, there is a need for better treatments for both primary and secondary stroke prevention. Design/Methods Genetic instruments for biomarkers were constructed by meta-analyzing GWAS pQTL results from the ORIGIN trial and 4 publicly available datasets (Suhre et al., 2017; Ahola et al., 2017; Folkersen et al., 2017; Sun et al., 2018), totalling 20,470 individuals and 3,249 biomarkers. GWAS summary statistics from MEGASTROKE were used in the analyses of cardioembolic, large artery, and small vessel stroke, and SiGN was used in the analysis of cryptogenic stroke. To elucidate the effects of significant biomarkers on health and disease beyond risk of stroke, phenome-wide analysis was conducted for all nondietary related traits in the UK-biobank study. Results Several significant biomarkers emerged for cardioembolic stroke (ABO, F11, SCARA5, TNFSF12) and large artery disease (ABO, LPA). No significant biomarkers were found for small-vessel disease and cryptogenic stroke after correcting for multiple hypotheses. Conclusions We identified potentially causal mediators for cardioembolic stroke and large-artery disease. Further investigation is warranted to determine whether these targets are amenable to pharmacologic intervention.

Contact Email: michael.chong@phri.ca

Disclosure and Study Support: None.

\section{Genetic influences on early neurological instability after acute ischemic stroke}

Laura Ibanez, PhD, ${ }^{1}$ Laura Heitch, $M D,{ }^{1}$ Caty Carrera, $M D,{ }^{2}$ Daniel Strbian, $M D, P h D,{ }^{3}$ Agnieszka Slowik, $M D, P h D,{ }^{4}$ Antonio Arauz, MD, PhD, ${ }^{5}$ Israel Fernandez, $P h D,{ }^{2}$ Joan Montaner, MD, PhD, Jin-Moo Lee, MD, PhD, ${ }^{1}$ and Carlos Cruchaga, $P h D^{1}$

${ }^{1}$ Washington University School of Medicine, Saint Louis, $\mathrm{MO}{ }^{2}$ Vall d'Hebron Institute of Research, Barcelona, Spain; ${ }^{3}$ Helsinki University Hospital, Finland; ${ }^{4}$ Jagiellonian University Medical College, Krakow, Poland; ${ }^{5}$ Instituto Nacional de Neurologia y Neurocirurgia, Tlalpan, Mexico.

Objective To identify the genetic loci related to early neurologic changes after ischemic stroke using a genome wide association study approach. Background Following acute ischemic stroke (AIS) onset, neurologic deficits can be highly unstable. Early neurologic change within the first 24 hours, defined here as $\Delta$ NIHSS24h (NIHSS baseline - NIHSS 24 hours), substantially influences long-term outcome. While several mechanisms, such as hemorrhagic transformation and recanalization, may impact $\Delta$ NIHSS24h, little is known about genetic influences. Design/Methods AIS patients were prospectively enrolled between 2008-2018 at 5 sites (St Louis, Barcelona, Helsinki, Krakow and Mexico). NIHSS scores were obtained within 6 hours and again at 24 hours after stroke onset. Genome-wide genotyping was generated for rare and common variants, imputing up to 6 million single nucleotide polymorphisms (SNPs) for all subjects $(\mathrm{N}=3,881)$. $\Delta$ NIHSS24h was used as a quantitative trait to measure early improvement/deterioration. Genome-wide complex trait analysis (GCTA) was performed. An association model was done, using NIHSS baseline, age, sex, PCA1 and PCA2 as covariates. All samples were analyzed separately using Plink1.9. Then, we used Mantra to perform a trans-ethnic meta-analysis. Results GCTA indicates that common variants account for $9 \%-11 \%$ of $\Delta$ NIHSS24h variability. We found 4 GWAs hits in the trans-ethnic meta-analysis. The rs 1900342 located within the LRRTM4 gene (Bayesian Factor $[\mathrm{BF}]=$ 5.038), the rs7562674 in METTL8 (BF = 5.327), rs2591460 in CYFIP2 (BF = 7.031) and rs11666496 within the NRLP genes region $(\mathrm{BF}=7.480)$. Conclusions Early neurologic 


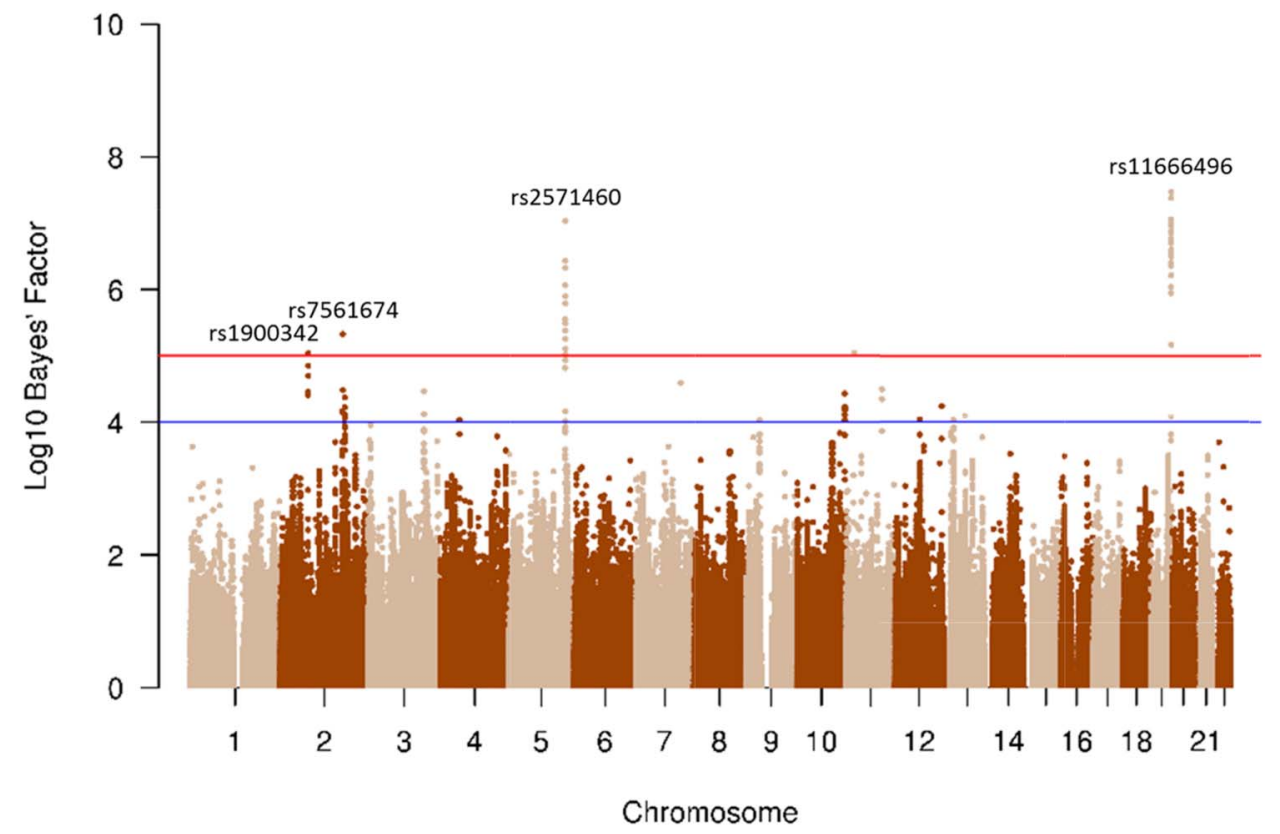

outcome after AIS is strongly influenced by genetics. The loci identified in this analysis are mostly related to inflammation, specially the top hit on chromosome 19 containing the NLRP genes, suggesting that inflammation is not only important for stroke but also influences early neurologic changes.

Contact Email: ibanezl@wustl.edu

\section{Disclosure and Study Support: NA.}

\section{Investigating the causal role of haematological traits on stroke and its subtypes}

Eric L. Harshfield, PhD, ${ }^{1}$ Matthew Sims, ${ }^{2}$ William J. Astle, PhD, ${ }^{1}$ Matthew Traylor, PhD, ${ }^{1}$ Willem H. Ouwehand, FMedSci, ${ }^{2}$ and Hugh S. Markus, $\mathrm{MD}^{1}$

${ }^{1}$ Stroke Research Group, Department of Clinical Neurosciences, University of Cambridge, Cambridge, UK; ${ }^{2}$ Department of Haematology, University of Cambridge and National Health Service Blood and Transplant, Cambridge, UK.

Objective To identify haematological traits with a causal role in the development of stroke and stroke subtypes. Background Blood cells and coagulation factors have many important roles including oxygen transport, immune response, iron homeostasis, clearance of apoptotic cells and toxins, vascular and endothelial cell function, response to systemic stress, and haemostasis. A recent analysis found evidence of a causal effect of several blood cell traits on coronary heart disease and Type 2 diabetes, but the causal relevance of haematological traits for stroke and its subtypes has not been established. This information is critical to inform the development of novel treatments for stroke patients. Design/ Methods We conducted a literature review of genome-wide association studies of haematological traits and downloaded publicly available summary statistics from these studies and the MEGASTROKE consortium. Mendelian randomization analyses were used to examine whether haematological traits have a causal effect on the development of stroke and its subtypes in both European-only and trans-ethnic populations. A gene score constructed from independent genetic variants associated with each trait was used as an instrumental variable to obtain an estimate of the causal effect using the ratio, inverse-variance weighted, weighted median, and MR-Egger approaches. Results The literature review identified 36 blood cell traits (platelets, mature/immature red cells, and myeloid/ lymphoid/compound white cells), 43 coagulation factors, and 3 markers of platelet function from 28 published GWAS, which included data from UK Biobank, INTERVAL, CHARGE Consortium, CARe Consortium, BioBank Japan Project, ARIC, Rotterdam Study, Framingham Heart Study, and other cohorts, involving over 350,000 individuals. Genetic associations with stroke and stroke subtypes were available in 67,162 cases and 454,450 controls. Preliminary analyses on a subset of traits showed that plateletcrit had a significant causal effect $\left(p<8.4746 \times 10^{-4}\right)$ on all stroke (AS), any ischaemic stroke (AIS), and cardioembolic stroke (CES) in both European-only and trans-ethnic populations; Factor VIII activity had a significant causal effect on AS, AIS, large-artery stroke (LAS), and CES in both populations; and von Willebrand Factor had a significant causal effect on AS and AIS in both populations and a marginally significant effect $(p<$ $1 \times 10^{-3}$ ) on CES in a trans-ethnic population. There were also suggestive causal associations of 27 of the blood cell traits on stroke and various subtypes; of activated partial thromboplastin time and Factor VII clotting activity with AS, AIS, and small vessel stroke (SVS); and of prothrombin time and collagen lag 
time with CES. Conclusions Our preliminary analyses have so far identified one blood cell trait, 2 coagulation factors, and one marker of platelet function that exhibit evidence of a significant causal association with stroke and various subtypes of stroke, as well as numerous haematological traits that are marginally significant or suggestive of a causal effect. Further investigation of these traits could lead to the development of novel drug targets for the treatment and prevention of stroke.

Contact Email: eh457@medschl.cam.ac.uk

Disclosure and Study Support: The MEGASTROKE project received funding from sources specified at megastroke. org/acknowledgments.html.

\section{Genetic characterization of embolic stroke of undetermined source: Whole exome sequencing of two Finnish families}

A. Ilinca A, MD, ${ }^{1,7}$ N. Martinez-Majander, $M D, M S c,{ }^{2} K$. Truvé, $\mathrm{PhD},^{3}$ S. Samuelsson, $\mathrm{MD}, \mathrm{PhD},{ }^{4} \mathrm{M}$. Soller, $\mathrm{MD}, \mathrm{PhD},{ }^{5} \mathrm{U}$. Kristoffersson, $\mathrm{MD}, \mathrm{PhD},{ }^{4} \mathrm{~T}$. Tatlisumak, $\mathrm{MD},{ }^{6} \mathrm{~J}$. Putaala, $\mathrm{MD},{ }^{2}$ and $A$. Lindgren, $M D, P h D^{1,7}$

${ }^{1}$ Department of Clinical Sciences Lund, Neurology, Lund University, Sweden; ${ }^{2}$ Department of Neurology, Helsinki University Hospital, Finland; ${ }^{3}$ Bioinformatics Core Facility, Sahlgrenska Academy at University of Gothenburg, Sweden; ${ }^{4}$ Department of Clinical Genetics and Pathology, Laboratory Medicine, Region Skane and Lund University, Sweden; ${ }^{5}$ Department of Clinical Genetics, Karolinska University Hospital, Solna, Sweden; ${ }^{6}$ Department of Clinical Neuroscience, Institute of Neuroscience and Physiology, Sahlgrenska
Academy at the University of Gothenburg, Sweden; ${ }^{7}$ Department of Neurology and Rehabilitation Medicine, Neurology, Skåne University Hospital, Lund, Sweden.

Objective To analyze the possibility of monogenic causes underlying stroke in young patients with ESUS. Background Embolic stroke of undetermined source (ESUS), defined as non-lacunar cryptogenic ischemic stroke in whom embolism from proximal sources is the likely stroke mechanism is not known to be associated with any monogenic condition. We studied 2 larger Finnish families with clustering of early-onset stroke, the probands experienced ESUS at the age of 31 and 48. Design/Methods Clinical data of 13 affected members from 2 families and 5 healthy family members were compiled. Whole exome sequencing (WES) of the 18 individuals was performed on Ion Proton (Life Technologies), using base and variant calling by Torrent Suite. Ion Reporter Libraries were generated with Ion AmpliSeq Exome RDY kit. Conventional filtering methods were used, considering only exonic or splice-site variants with a minimum allele frequency $<1 \%$. Further, we used our recently published comprehensive stroke gene panel containing all the known ischemic strokerelated genetic to date. Finally, we considered co-segregation, assuming an autosomal dominant $(\mathrm{AD})$ model of inheritance of the underlying disease. Results In family \#1, 7 family members had experienced stroke, with 6 of them in a model compatible with an $\mathrm{AD}$ condition. Eleven of these family members were genetically analyzed by WES. Of them, 3 were younger than 49 years when diagnosed with stroke with 2 of them fulfilling ESUS criteria. In family \#2, 5 persons had experienced stroke, of which 3 occurred at age younger than 49 in 3 consecutive generations. ESUS was identified in 2 individuals. Co-segregation analysis identified 3 nonsynonymous single nucleotide variants of unknown significance in 2 different genes in family \#1 and 3 variants in 3 different genes in family \#2 (table). Conclusions Further

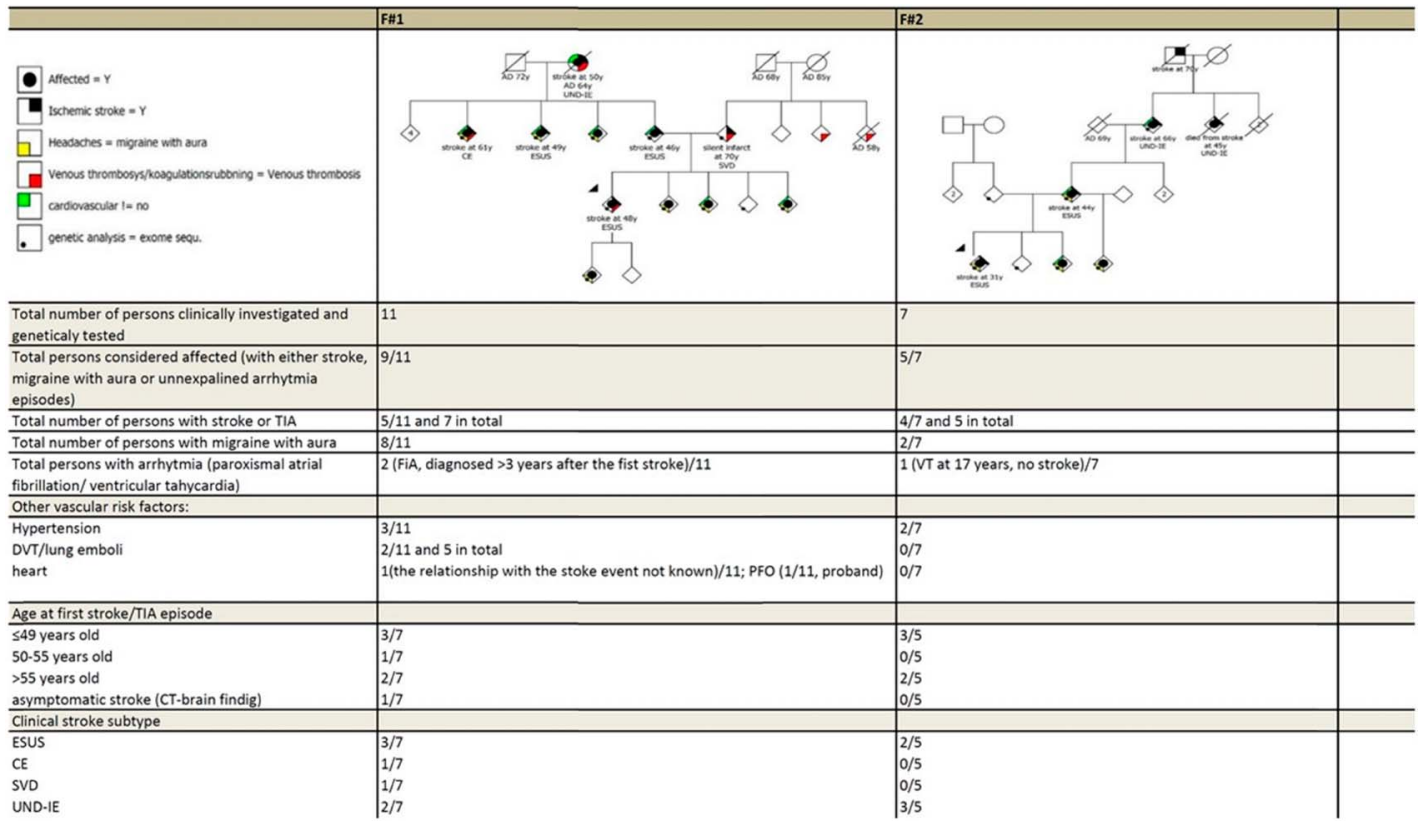


genetic analyses may identify the gene(s) responsible for monogenic inheritance of cryptogenic stroke in these families.

Contact Email: andreea.ilinca@med.lu.se

Disclosure and Study Support: None.

\section{Plasma-proteomic and} metabolomic profiles in ischemic stroke: Towards the identification of non-invasive biomarkers

A. Donatti, PhD, ${ }^{1}$ A.M. Canto, PhD, ${ }^{1}$ A.A.V. Oliveira e Sousa, $M D,^{2}$ W.M. Avelar, MD, ${ }^{2}$ R.A.S. Robinson, PhD, ${ }^{3}$ M. Quintero, $P h D,{ }^{4}$ L. Tasic, $P h D,{ }^{4}$ and I. Lopes-Cendes, $M D, P h D^{1}$

${ }^{1}$ Department of Medical Genetics, University of Campinas (UNICAMP), Campinas-Brazil; ${ }^{2}$ Department of Neurology; School of Medical Science, and the Brazilian Institute of Neuroscience and Neurotechnology (BRAINN), University of Campinas (UNICAMP), Campinas-Brazil; ${ }^{3}$ Department of Chemistry, Vanderbilt University, Nashville, TN; ${ }^{4}$ Department of Organic Chemistry, Institute of Chemistry. University of Campinas (UNICAMP), Campinas-Brazil.

Objective We report here results from a pilot study. Overall, we aim to identify non-invasive, circulating biomarkers for ischemic stroke (IS). Background Biomarkers are small molecules, altered during the disease process and that can be used to improve diagnosis and/or to determine prognosis, including response to treatment. Proteins and small molecules, metabolites, have the potential to be useful biomarkers due to their ability to reflect the molecular mechanism underlying disease. Design/Methods In this initial study we evaluated plasma samples from patients in the acute phase of IS, samples from patients in the chronic phase of IS and samples from healthy, age-matched controls $(n=21)$. For the proteomics analysis samples were digested with acetone and albumin fractions were separated by $12 \%$ SDS-page. The gel was fragmented in 2 different parts: one containing albumin and other with smaller proteins. Both fractions were purified and sent for analysis on a mass spectrometer nanoLC PROXEON EASY-nLCII/ Orbitrap Elite Hybrid Ion trap Mass Spectrometer ${ }^{\circledR}$ (Thermo Fisher). Biological pathways were identified by the Metacore ${ }^{\circledR}$ software. For the metabolomic study samples were evaluated using $1 \mathrm{H}$ NMR spectra, recorded under the same conditions in a $600 \mathrm{MHz}$ spectrometer (Bruker). Data processing and interpretation were performed using a combination of liquid-state NMR techniques and chemometrics analysis. Results We found an overall reduction of $14 \%$ on the total amount of proteins identified in samples collected in the acute phase of IS. In addition, we identified 127 proteins abnormally regulated in the acute phase of $\mathrm{IS}$; these include CFB, COX17, APOD, APOE, and HPX. A preliminary evaluation of the metabolite profiles revealed stronger signals in samples from patients in the acute phase of IS at spectral regions $\delta 8.20, \delta$ $8.25, \delta 8.40$ and $\delta 8.50$. These signals are mainly related to aromatic and acid hydrogen atoms. Conclusions Our proof-ofconcept preliminary study showed that we could identify proteins which are abnormally regulated in the acute phase of IS, these were mainly related to inflammatory and lipid metabolism pathways. In addition, we observed distinct metabolic signatures in plasma samples of patients in the acute phase of IS, which are probably metabolites from the mitochondrial oxidation of fatty acids and DNA damage repair.

Contact Email: icendes@unicamp.br

Disclosure and Study Support: Study supported by BRAINN, FAPESP-Brazil.

\section{Circular RNAs associated with kinase activity at synaptic regions exhibit differential expression in penumbra}

Elaine Huang, $\mathrm{PhD},{ }^{1} \mathrm{Dan} \mathrm{Lu}, \mathrm{MD},{ }^{2,3}$ Chi Kwan Tsang, $\mathrm{PhD},{ }^{3}$ Anding $\mathrm{Xu}, \mathrm{MD},{ }^{2,3}$ and Eric S. Ho, $\mathrm{PhD}^{4}$

${ }^{1}$ Neuroscience Program, Lafayette College, Easton, PA; ${ }^{2}$ Department of Neurology and Stroke Center, the First Affiliated Hospital, Jinan University, Guangzhou, China; ${ }^{3}$ Clinical Neuroscience Institute, The First Affiliated Hospital of Jinan University, Guangzhou, China; ${ }^{4}$ Department of Biology, Lafayette College, Easton, PA.

Objective To identify differentially expressed circular RNAs in neuronal tissues after acute ischemic stroke. Background Circular RNAs (circRNAs) are synthesized via back-splicing, the process in which a downstream splice acceptor or 5' splice site (ss) covalently joins an upstream donor or $3^{\prime}$ ss. These molecules were first characterized in 1993 but mostly regarded as mis-spliced products until the recent decade. With the advent of high throughput DNA sequencing technology, circRNAs are now detected routinely in diverse species, including animals and plants. In humans, the median length of circRNAs is $800 \mathrm{bps}$. While the functions of most circRNAs are unknown, a handful have been identified as miRNA sponges. Such findings may suggest their roles in gene regulation. Not only are circRNAs highly enriched in brain tissue, but levels of circRNA have also been found to be significantly altered in the core region after stroke, suggesting functionality in neural response to stroke injury. As such, we are interested in studying the impact of ischemic stroke on circRNA expression in the penumbra of mice. Design/Methods Focal ischemia was induced by intraluminal middle cerebral artery occlusion (MCAO) for 3 hours. Total RNA was harvested from tissues removed from penumbra and control regions for RNA-Seq analysis. Short reads were mapped to 
the mouse genome (mm10) by STAR and BWA. circRNAs were detected computationally by CIRCexplorer2 and CIRI2. Two thousand seven hundred forty-six and 2258 circRNAs were identified in the control region and penumbra respectively by both algorithms and in all 3 replicates for each brain region. Expression profiles of circRNAs from penumbra and control were compared using edgeR. Functional enrichment analysis using DAVID was performed on the host genes of circRNAs that exhibited a twofold change or greater between the control and penumbra regions with $p<0.05$. Results Between the control and penumbra region, 745 differentially expressed circRNAs were detected from 624 genes. Based on the top 5 functional clusters generated by DAVID, these genes are involved with synaptic regions, kinase activity/ phosphorylation, intracellular signaling, metal ion binding, and C 2 calcium-dependent targeting. Conclusions We have identified hundreds of differentially expressed circRNAs in penumbra vs control in mice. Genes encoding these circRNAs are associated with activity at cell junctions, in addition to intracellular signaling and protein phosphorylation control. Since neuron stimulation has been a major after stroke therapeutic strategy, future research on penumbra-enriched or depleted circRNAs may be key for the development of novel treatments.

Contact Email: hoe@lafayette.edu

Disclosure and Study Support: None.

\section{Depressive symptoms and recovery after ischemic stroke: Mendelian randomization analysis of GISCOME data}

Dipender Gill, MD, ${ }^{1}$ Jane M. Maguire, $P h D^{2,3,4}$; on behalf of investigators from the GISCOME collaboration of the ISGC

${ }^{1}$ Department of Epidemiology and Biostatistics, Imperial College London, United Kingdom; ${ }^{2}$ Faculty of Health, University of Technology, Australia; ${ }^{3}$ Hunter Medical Research Institute, University of Newcastle, Australia; ${ }^{4}$ Priority Research Centre for Stroke and Traumatic Brain Injury, University of Newcastle, Australia.

Objective To investigate the effect of depressive symptoms on outcomes after ischemic stroke using Mendelian Randomization (MR). Background Depressive symptoms have been associated with worse outcomes in a range of disease settings. However, evidence in this area is largely restricted to observational study, where spurious associations may arise due to reverse causation or confounding from unknown and unmeasured factors. The MR technique overcomes these limitations by using genetic variants to instrument the effect of an exposure. Design/Methods A 2-sample MR approach using summary data was used. Instruments for depressive symptoms were identified from a genome-wide association study (GWAS) meta-analysis of 354,862 individuals, with corresponding genetic association estimates for recovery after ischemic stroke taken from a GWAS meta-analysis of 5,762 patients. Statistical power calculation was performed prior to analysis. Fixed-effects inverse variance weighted (IVW) MR was used in the main analysis, with heterogeneity estimates, weighted median MR and MR-PRESSO used to explore possible bias relating to inclusion of pleiotropic SNPs. Results Power calculations suggested that with the available data, $80 \%$ statistical power would be achieved with a minimum detectable odds ratio (OR) of 1.7 for a modified Rankin Scale of 3-6 rather than 0-2 per standard deviation (SD) increase in depressive symptoms. The main IVW MR analysis demonstrated a detrimental effect of depressive symptoms on functional recovery after ischemic stroke (OR $9.27,95 \%$ CI 1.39 to $61.99, p=0.02$ ). There was no evidence of heterogeneity between MR estimates produced by individual SNPs (I2 0\% [95\% CI 0\%-32\%], Cochran's Q $p=$ 0.67). Comparable MR estimates were obtained when using weighted median MR (OR 19.33, 95\% CI 1.13 to 329.64, $p=$ 0.04 ) or MR-PRESSO (OR 9.27, 95\% CI 1.59 to 54.21, $p=$ 0.02 , with no outliers detected), which are more robust to inclusion of pleiotropic variants. Conclusions Despite the limited statistical power of this analysis, we were able to demonstrate MR evidence of a causal effect of depressive symptoms on functional recovery after ischemic stroke. Further work is required to explore the clinical implications of these findings, and whether depressive symptoms may be therapeutically targeted to improve recovery in stroke victims.

Contact Email: dipender.gill@imperial.ac.uk

Disclosure and Study Support: None.

\section{Intracerebral hemorrhage induces inflammatory gene expression in swine: A study of RNA-seq in mononuclear cells}

Kyle B. Walsh, MD, MS, ${ }^{1,2}$ Xiang Zhang, $P h D,{ }^{3}$ Xiaoting Zhu, $M S,{ }^{4}$ Eric Wohleb, PhD, ${ }^{5,7}$ Daniel Woo, MD, MS, ${ }^{1,6}$ Long Lu, $\mathrm{PhD},{ }^{4}$ and Opeolu Adeoye, $\mathrm{MD}, \mathrm{MS}^{1,2}$

${ }^{1}$ University of Cincinnati Gardner Neuroscience Institute, Cincinnati, $\mathrm{OH} ;{ }^{2}$ Department of Emergency Medicine, University of Cincinnati, $\mathrm{OH} ;{ }^{3}$ Department of Environmental Health, University of Cincinnati, OH; ${ }^{4}$ Division of Biomedical Informatics, Cincinnati Children's Research Foundation, Cincinnati, OH, USA; 5. Department of Pharmacology and Systems Physiology, University of Cincinnati, Cincinnati, $\mathrm{OH}$, USA; 6. Department of Neurology and Rehabilitation Medicine, University of Cincinnati, Cincinnati, OH, USA; 7. 
University of Cincinnati Neurobiology Research Center, Cincinnati, $\mathrm{OH}$.

Objective To perform RNA-seq and bioinformatics enrichment analysis in peripheral blood mononuclear cells (PBMCs) from a swine model of intracerebral hemorrhage (ICH). Background Intracerebral hemorrhage (ICH) is a severe neurologic disorder with no proven treatment. Our prior research identified a significant association with higher monocyte level and greater ICH mortality. Although there is substantial interest in neuroinflammation as a therapeutic target following $\mathrm{ICH}$, these processes remain poorly understood. To advance our understanding, we sought to identify the gene expression changes after $\mathrm{ICH}$ using a swine model to test the hypothesis that $\mathrm{ICH}$ would induce changes in PBMC gene expression. Design/Methods In 10 pigs with autologous blood injection to induce ICH, 2 PBMC samples were drawn from each pig with the first immediately prior to $\mathrm{ICH}$ induction and the second 6 hours later. RNA-seq was performed to identify differentially expressed genes (DEGs) and associated Gene Ontology (GO) and Kyoto Encyclopedia of Genes and Genomes (KEGG) pathways. Results There were 182 significantly upregulated and 153 significantly down-regulated DEGs after ICH. Consistent with findings in humans, significant GO and KEGG pathways were those primarily related to inflammation, immune response, and response to infectious pathogens. There were 5 genes, all with increased expression post- $\mathrm{ICH}$, that were repeatedly identified as significant DEGs in the statistically significant KEGG pathways: CD14 (cluster of differentiation 14), TLR4 (toll-like receptor-4), CXCL8 (CXC motif chemokine-8), IL-18 (interleukin-18), and CXCL2 (CXC motif chemokine-2). Conclusions $\mathrm{ICH}$ induced changes in PBMC gene expression within 6 hours of onset. DEGs that were highly expressed in the significant biological pathways included those related to inflammation, the immune response, and, more specifically, monocyte activation. Further research is needed to determine if these changes affect outcomes and may represent new therapeutic targets.

Contact Email: walshk4@uc.edu

Disclosure and Study Support: The financial support for blood sample collection, processing, storage, RNA sequencing, and bioinformatics analysis was provided by the University of Cincinnati Gardner Neuroscience Institute. The financial support for the swine experiments only was provided by Sense Diagnostics, LLC. The research reported in the submitted abstract was performed as a sub-study to a swine intracerebral hemorrhage study performed by Sense Diagnostics for the testing of a non-invasive brain monitor. The reported results in this abstract, and the RNA-seq data overall, have no bearing on the development of this non-invasive brain monitoring device. The author Opeolu Adeoye MD, MS, holds equity in Sense Diagnostics, LLC. All other authors have no disclosures or conflicts of interest to report.

\section{Update on genomics of intracerebral hemorrhage}

Christopher Anderson, $M D,{ }^{1}$ Daniel Woo, $M D,{ }^{2,3}$ Guido Falcone, $M D,{ }^{4}$ and Jonathan Rosand, $M D^{1}$

${ }^{1}$ Department of Neurology, Massachusetts General Hospital, Boston; ${ }^{2}$ University of Cincinnati Gardner Neuroscience Institute, $\mathrm{OH} ;{ }^{3}$ Department of Neurology and Rehabilitation Medicine, University of Cincinnati, OH; ${ }^{4}$ Department of Neurology, Yale University Hospital, New Haven, CT.

Objective and Background The ISGC intracerebral hemorrhage ( $\mathrm{ICH})$ working group has a number of major projects underway and future collaborative efforts planned. Design/Methods The Genetic and Environmental Risk Factors for Hemorrhagic Stroke (NS036695) study is a major multi-center study to perform RNA-sequencing and deep sequencing of the Chr1q22 locus associated with non-lobar ICH. With a goal of 1000 total cases, the study has recruited 535 as of October 26, 2018. The Ethnic/Racial Variations of Intracerebral Hemorrhage-Longitudinal (NS093870) study seeks to perform long-term follow-up of 900 cases of the ERICH study and perform cause of death, subsequent stroke and significant events assessment as well as cognitive evaluations. The study has identified 937 cases for follow-up with a mean follow-up of 4.8 years of which 732 are still in active follow-up. The SAFARI-ICH (NS103924) study is performing annotation and association testing at 3 established risk loci in over 4,000 ICH cases with whole genome sequencing, seeking to define causal variation and establish cellular model systems for translational investigation. A parallel effort is using machine learning to automatically ascertain acute neuroimaging biomarkers in CT scans from patients enrolled in the aforementioned studies (AG059992). Conclusions These efforts will characterize the genetic underpinnings of primary and secondary brain injury in ICH. We further propose a large-scale multi-ethnic genome wide association study combining any and all available samples from around the world and invite participation and collaboration.

\section{Contact Email: WOODL@UCMAIL.UC.EDU}

Disclosure and Study Support: None.

\section{Genome-wide association study of cerebral small vessel disease identifies novel loci at 2q33 and 13q34}

Jaeyoon Chung, PhD ${ }^{1,2}$, Sandro Marini, $M D^{1,2,3}$, Matthew Traylor $^{4}$, Rainer Malik ${ }^{5}$, Carl D. Langefeld 6 , Daniel $\mathrm{Woo}^{7}$, Jonathan Rosand, $M D, M S c^{1,2,3}$, and Christopher D Anderson, $M D, M M S c^{1,2,3}$; on behalf of the International Stroke Genetics Consortium 
${ }^{1}$ Center for Genomic Medicine, Massachusetts General Hospital, Boston; ${ }^{2}$ Program in Medical and Population Genetics, Broad institute, Boston, MA; ${ }^{3}$ Department of Neurology, Massachusetts General Hospital, Boston; ${ }^{4}$ Department of Clinical Neurosciences, University of Cambridge, UK; ${ }^{5}$ Institute for Stroke and Dementia Research, University Hospital, LMU Munich, Germany; ${ }^{6}$ Center for Public Health Genomics and Department of Biostatistical Sciences, Wake Forest School of Medicine, Winston-Salem, NC; ${ }^{7}$ Department of Neurology and Rehabilitation Medicine, University of Cincinnati College of Medicine, $\mathrm{OH}$.

Objective Cross-phenotype analysis of Intracerebral hemorrhage (ICH) and small vessel ischemic stroke (SVS) will improve statistical power to detect novel genetic risk factors for cerebral small vessel disease (CSVD), elucidating underlying disease mechanisms that may form the basis for future treatments. Background Intracerebral hemorrhage (ICH) and small vessel ischemic stroke (SVS) are the most severe manifestation of cerebral small vessel disease (CSVD) with no established preventive approaches beyond hypertension management. Design/Methods We performed GWAS for 1,005 non-lobar ICH and 1,711 stroke-free controls after imputation using HRC reference panels. Non-lobar ICH GWAS results were meta-analyzed with GWAS summary statistics for SVS from MEGASTROKE, using "MultiTrait Analysis of GWAS" (MTAG) to integrate genetic associations across traits. Tissue-specific gene-level associations were generated by S-PrediXcan using the MTAG result of non-lobar ICH. For variants with genome-wide significant (GWS; $p<5 \times 10^{-8}$ ) association from MTAG, we further evaluated potential regulatory functions and association with transcript-level expression (i.e., eQTL) using HaploReg and GTEx databases. We also explored cell type-specific expressions for the genes at GWS loci via human brain single cell RNA-Seq. Results After combining GWAS datasets using MTAG, our functional sample size increased to 241,024 participants (6,255 ICH/SVS cases and 234,769 controls). GWS associations were observed for the non-lobar ICH in the MTAG result with rs2758605 at 1q22; rs72932727 at 2q33; and rs9515201 at 13q34. According to GTEx, rs2758605 (1q22), rs72932727 (2q33) and rs9515201 (13q34) are significant cis-eQTLs for PMF1 ( $p=1 \times 10^{-4}$ in nerve), NBEAL1, ICA1L, and CARF ( $p<7.7 \times 10^{-7}$ in artery) and COL4A2/COL4A1 ( $p<0.008$ in thyroid), respectively. From the gene-based association test by S-PrediXcan, 2 genes attained GWS level: ICA1L at $2 \mathrm{q} 33$ in tibial nerve $(p=1.3 \times$ $\left.10^{-6}\right)$ and $\mathrm{ZCCHC} 14$ at $16 \mathrm{q} 24$ in tibial artery $(p=2.2 \times$ $\left.10^{-6}\right)$. Brain cell-type specific expression profiling found that PMF1 at $1 \mathrm{q} 22$ and COL4A1 and COL4A2 at 13q34 were mainly expressed in endothelial cells, while the genes at 2q33-ICA1L, CARF, and NBEAL1-were expressed in various cells: astrocytes, oligodendrocytes, and neurons. Conclusions Our cross-phenotype study of non-lobar ICH and SVS reveals 2 novel CSVD loci: 2 q33 and 13q34. We have begun to replicate these associations and to identify functional causal variants in independent whole genome sequencing studies of ICH.

Contact Email: jchung22@mgh.harvard.edu

Disclosure and Study Support: None. 


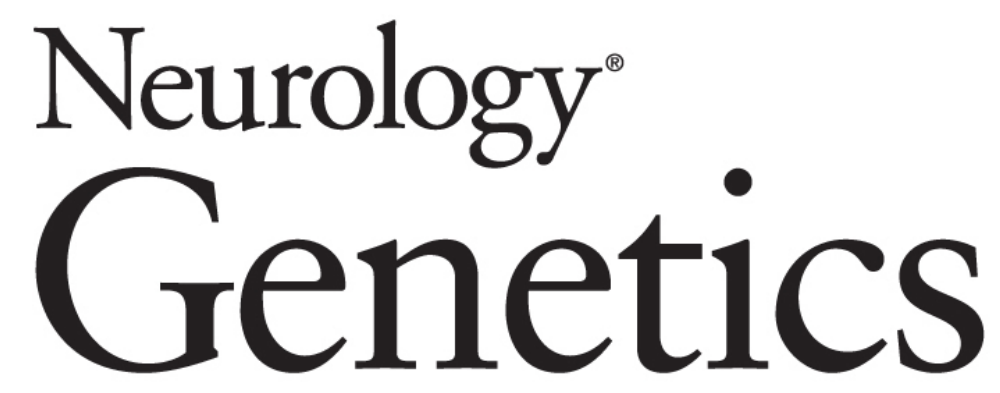
Proceedings of the 24th International Stroke Genetics Consortium Workshop: Washington, DC, USA
Paul A. Nyquist and John W. Cole Neurol Genet 2019;5;
DOI 10.1212/NXG.0000000000000325

This information is current as of April 8, 2019

\section{Updated Information \& Services}

Permissions \& Licensing

\section{Reprints}

including high resolution figures, can be found at: http://ng.neurology.org/content/5/1_Supplement_1/e325.full.html

Information about reproducing this article in parts (figures,tables) or in its entirety can be found online at:

http://ng.neurology.org/misc/about.xhtml\#permissions

Information about ordering reprints can be found online: http://ng.neurology.org/misc/addir.xhtml\#reprintsus

Neurol Genet is an official journal of the American Academy of Neurology. Published since April 2015, it is an open-access, online-only, continuous publication journal. Copyright ( 2019 American Academy of Neurology. All rights reserved. Online ISSN: 2376-7839.

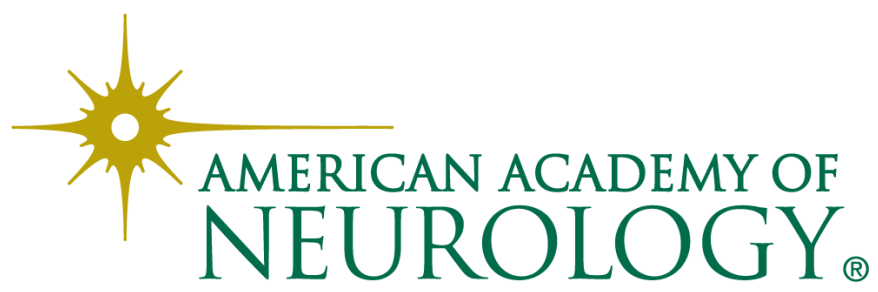

